\title{
Quasipolynomial formulas for the Kronecker coefficients indexed by two two-row shapes (extended abstract)
}

\author{
Emmanuel Briand 1 " Rosa Orellana ${ }^{2}$ and Mercedes Rosas 1 ": \\ ${ }^{1}$ Departamento de Álgebra, Facultad de Matemáticas, Aptdo. de Correos 1160, 41080 Sevilla, Spain. \\ 2 Dartmouth College, Mathematics Department, 6188 Kemeny Hall, Hanover, NH 03755, USA.
}

\begin{abstract}
We show that the Kronecker coefficients indexed by two two-row shapes are given by quadratic quasipolynomial formulas whose domains are the maximal cells of a fan. Simple calculations provide explicitly the quasipolynomial formulas and a description of the associated fan.
\end{abstract}

These new formulas are obtained from analogous formulas for the corresponding reduced Kronecker coefficients and a formula recovering the Kronecker coefficients from the reduced Kronecker coefficients.

As an application, we characterize all the Kronecker coefficients indexed by two two-row shapes that are equal to zero. This allowed us to disprove a conjecture of Mulmuley about the behavior of the stretching functions attached to the Kronecker coefficients.

Résumé. Nous démontrons que les coefficients de Kronecker indexés par deux partitions de longueur au plus 2 sont donnés par des formules quasipolynomiales quadratiques dont les domaines de validité sont les cellules maximales d'un éventail. Des calculs simples nous donnent une description explicite des formules quasipolynomiales et de l'éventail associé. Ces nouvelles formulas sont obtenues de formules analogues pour les coefficients de Kronecker réduits correspondants et au moyen d'une formule reconstruisant les coefficients de Kronecker à partir des coefficients de Kronecker réduits.

Une application est la caractérisation exacte de tous les coefficients de Kronecker non-nuls indexés par deux partitions de longueur au plus deux. Ceci nous a permis de réfuter une conjecture de Mulmuley au sujet des fonctions de dilatations associées aux coefficients de Kronecker.

Keywords: Kronecker coefficients, internal product of symmetric functions, Saturation properties, Representations of the symmetric group

\footnotetext{
${ }^{\dagger}$ Supported by a Juan de la Cierva Fellowship (MICINN, Spain) and Projects MTM2007-64509 (MICINN, Spain) and FQM333 (Junta de Andalucia).

¥Supported by a Ramón y Cajal Fellowship (MICINN, Spain) and Projects MTM2007-64509 (MICINN, Spain) and FQM333 (Junta de Andalucia).
}

1365-8050 @ 2009 Discrete Mathematics and Theoretical Computer Science (DMTCS), Nancy, France 


\section{Introduction}

A fundamental problem in algebraic combinatorics is the Clebsch-Gordan problem: given a linearly reductive group $G$, give a combinatorial description of the coefficients $m_{\mu \nu}^{\lambda}$ in the decomposition into irreducibles of the tensor product of two (finite-dimensional complex) irreducible representation $V_{\mu}(G)$ and $V_{\nu}(G)$ :

$$
V_{\mu}(G) \otimes V_{\nu}(G) \cong \bigoplus_{\lambda} m_{\mu \nu}^{\lambda} V_{\lambda}(G)
$$

While this problem has been solved satisfactorily for the general linear group, $G L(n)$, the most elementary linear group, this is not the case for the symmetric group, $S_{n}$, the most fundamental finite group.

In the case of $G L(n)$, the coefficients $m_{\mu \nu}^{\lambda}=c_{\mu \nu}^{\lambda}$ are the well known Littlewood-Richardson coefficients. There exists several combinatorial descriptions for them. One of these descriptions was given by Berenstein and Zelevinsky (1992) that showed that $c_{\mu \nu}^{\lambda}$ counts the integral points in a well-defined family of polytopes. This initiated a series of works concerning the stretching functions associated to these coefficients that culminated with the proof by Knutson and Tao (1999) of the saturation conjecture. Finally, Rassart (2004) showed that the Littlewood-Richardson coefficients $c_{\mu \nu}^{\lambda}$ are given by polynomial functions of the parts of $\lambda, \mu$ and $\nu$, on the maximal cells of a fan.

For the symmetric group $S_{n}$, the coefficients $m_{\mu \nu}^{\lambda}=g_{\mu \nu}^{\lambda}$ are called the Kronecker coefficients. Amazingly, there is no combinatorial description of these coefficients in general. Particular families have been investigated. In this paper the Kronecker coefficients indexed by two two-row shapes are considered. They are the coefficients $g_{\mu \nu}^{\lambda}$ such that both $\mu$ and $\nu$ have two rows. Formulas for them have already been given by Remmel and Whitehead (1994) and Rosas (2001). Recent works by Luque and Thibon (2003); Garsia et al. (2008); Brown et al. (2008) have revived the interest of obtaining better formulas for the Kronecker coefficients indexed by two two-row shapes as Hilbert series related to these coefficients have been linked to problems in quantum information theory.

New problems about the Clebsch-Gordan coefficients have been raised recently by the specialists of computational complexity. Narayanan (2006) showed that the computation of the Littlewood-Richardson coefficients is a \#P-complete problem. Bürgisser and Ikenmeyer (2008) showed that the computation of the Kronecker coefficients is \#P-hard. On the other hand, the saturation property implies that the non-vanishing of a Littlewood-Richardson coefficient can be decided in polynomial time (Mulmuley and Sohoni 2005). Is it also the case for the Kronecker coefficients? This question lies at the heart of a detailed plan, Geometric Complexity Theory, that Mulmuley and Sohoni (2001) elaborated to prove that $P \neq N P$ over the complex numbers (an arithmetic, non-uniform version of $P \neq N P$ ). This lead Mulmuley (2007) to state a series of conjectures about the stretching functions associated to the Kronecker coefficients. The scarce information available about Kronecker coefficients made difficult even the experimental checking of these conjectures. By means of the formulas by Remmel and Whitehead (1994) and Rosas (2001) it was only possible to check them on large samples of Kronecker coefficients indexed by two two-row shapes (see Mulmuley, 2007).

The present article obtains a new description for the Kronecker coefficients indexed by two two-row shapes, given by quasi-polynomial functions on the chambers of fans, resembling the description of Rassart (2004) for the Littlewood-Richardson coefficients. It is efficient enough to check Mulmuley's conjectures for all Kronecker coefficients indexed by two two-row shapes (and, actually, disprove them by providing explicit counter-examples). We start our investigation by looking at Murnaghan's reduced Kronecker coefficients $\bar{g}_{\alpha \beta}^{\gamma}$ Murnaghan, 1938), a related family of coefficients indexed by triples of parti- 
tions, which are stable values of stationary sequences of Kronecker coefficients. Our first result expresses the Kronecker coefficients in terms of the reduced Kronecker coefficients (Theorem 3). Exploiting the work of Rosas (2001) we are able to show that the reduced Kronecker polynomials related to the two-row family count integral points in a polygon of $\mathbb{R}^{2}$. From this we describe an explicit piecewise quasipolynomial formula for these reduced Kronecker coefficients. The pieces are the 26 maximal cells of a fan. Last, using our formula that recovers the Kronecker coefficients from the reduced Kronecker coefficients, we obtain, with the help of the Maple package convex by Franz (2006), explicit piecewise quasipolynomial formulas for the Kronecker coefficients indexed by two two-row shapes. It is given by 74 quadratic quasipolynomials whose domains are the maximal cells of a fan.

As an application, we list all Kronecker coefficients indexed by two two-row shapes that are equal to zero. This made possible the discovery of counter-examples to Mulmuley's conjectures (Briand et al. 2008). In short, the advantage of our results is that for the first time we can completely study a complete nontrivial family of the Kronecker coefficients.

The detailed proofs will be presented in a full version (Briand et al., In preparation) of this extended abstract.

\section{Piecewise Quasipolynomials}

We now give a more detailed description of the main result. A quasipolynomial is a function on $\mathbb{Z}^{n}$ given by polynomial formulas, whose domains are the cosets of a full rank sublattice of $\mathbb{Z}^{n}$. Remarkable examples of (univariate) quasipolynomials are the Ehrhart functions of polytopes of $\mathbb{R}^{k}$ with rational vertices, that count the integral points in the dilations of the polytope (see Stanley, 1997, chap. 4).

We will obtain a description for the Kronecker coefficients indexed by two two-row shapes as a function of the following kind.

Definition $1 A$ vector partition-like function is a function $\phi$ on $\mathbb{Z}^{n}$ fulfilling the following: (i) There exists a convex rational polyhedral cone $C$ such that $\phi$ is zero outside $C$. (ii) Inside $C$, the function $\phi$ is given by quasipolynomial formulas whose domains are (the sets of integral points of) the maximal (closed) cells of a fan $\mathfrak{F}$.

If $C$ and $\mathfrak{F}$ are as above and $Q$ is the family of quasipolynomial formulas, indexed by the maximal cells of $\mathfrak{F}$, we say that the triple $(C, \mathfrak{F}, Q)$ is a presentation of $\phi$ as a vector partition-like function.

Remark 1 A sum of vector partition-like functions $\phi_{1}, \phi_{2}$ is not necessarily vector partition-like. It is, however, the case when the functions admit presentations $(C, \mathfrak{F}, Q)$ and $\left(C^{\prime}, \mathfrak{F}^{\prime}, Q^{\prime}\right)$ with the same cone: $C=C^{\prime}$.

Examples of vector partition-like functions are the vector partition functions, whose corresponding fans are the chamber complexes (see Sturmfels, 1995; Brion and Vergne, 1997).

Vector partition-like functions also arise as functions counting integral solutions to some systems of linear inequalities depending on parameters. Precisely, consider a system of inequalities of the form

$$
u_{i}(x)+c_{i}(h) \geq 0, \quad i=1, \ldots, N
$$

where the functions $u_{i}$ and $c_{i}$ are integral, homogeneous linear forms on $\mathbb{R}^{m}$ and $\mathbb{R}^{n}$ respectively. The unknown is $x$ and the parameter is $h$. Assume that for any $h \in \mathbb{R}^{n}$ the set of solutions $x$ of the system is bounded. Let $h \mapsto \phi(h)$ be the function that counts the integral solutions $x$ of the system. This function $\phi$ 
is vector partition-like. This follows from the reduction of this function to a vector partition function (see Brion and Vergne, 1997). Here the cone $C$ in Definition 1 is the set of values of the parameter $h$ making the system feasible.

Let $\ell$ be a positive integer. The function $(\lambda, \mu, \nu) \mapsto c_{\mu, \nu}^{\lambda}$ from triples of partitions with at most $\ell$ parts to Littlewood-Richardson coefficients is vector partition-like. This is because this function counts the integral solutions of a system of inequalities depending on parameters (the parts of the partitions) of the form (17). Indeed, such a system can be derived from the Littlewood-Richardson rule (see Mulmuley and Sohoni 2005). Alternatively, one can use the system defining Knutson and Tao's Hive polytopes (see the exposition by Buch, 2000).

It is natural to ask if similar results also hold for the Kronecker coefficients. Let $\ell_{1}$ and $\ell_{2}$ be positive integers. If $\mu$ and $\nu$ are partitions of length at most $\ell_{1}$ and $\ell_{2}$ respectively then $g_{\mu, \nu}^{\lambda}$ can be nonzero only if $\lambda$ has at most $\ell_{1} \ell_{2}$ parts. The analogous function to consider is thus $G_{\ell_{1}, \ell_{2}}:(\lambda, \mu, \nu) \mapsto g_{\mu, \nu}^{\lambda}$ defined on triples of partitions with at most $\ell_{1} \ell_{2}, \ell_{1}$ and $\ell_{2}$ parts respectively. No interpretation of the functions $G_{\ell_{1}, \ell_{2}}$ as counting integral solutions to systems of inequalities of the form (1) is known. Nevertheless, very close results were obtained by Mulmuley (2007): (i) The functions $G_{\ell_{1}, \ell_{2}}$ fulfill the conditions in Definition 1 with $\mathfrak{F}$ a complex of polyhedral cones instead of a fan. (ii) For any $\lambda, \mu, \nu$, the stretching function $N \in \mathbb{N} \mapsto g_{N \mu, N \nu}^{N^{\lambda}}$ is a univariate quasipolynomial. Here $N \lambda$ stands for the partitions obtained from $\lambda$ by multiplying all parts by $N$. Combining these two results, one gets that the functions $G_{\ell_{1}, \ell_{2}}$ fulfill the conditions in the definition of vector partition-like with "maximal closed cells" replaced with "open cells" in (ii).

The simplest non-trivial case is $G_{2,2}$, describing the Kronecker coefficients indexed by two two-row shapes. Even this case is somehow difficult. In this work we prove the following:

Theorem 1 The function

$$
G_{2,2}:\left(\lambda_{1}, \ldots, \lambda_{4}, \mu_{1}, \mu_{2}, \nu_{1}, \nu_{2}\right) \in \mathbb{Z}^{8} \mapsto g_{\left(\mu_{1}, \mu_{2}\right)\left(\nu_{1}, \nu_{2}\right)}^{\left(\lambda_{1}, \lambda_{2}, \lambda_{3}, \lambda_{4}\right)}
$$

is vector partition-like.

Remark 2 A Kronecker coefficient $g_{\mu, \nu}^{\lambda}$ can be nonzero only if its three indexing partitions have the same weight. This and the formula $g_{\left(\mu_{1}, \mu_{2}\right)\left(\nu_{1}, \nu_{2}\right)}^{\left(\lambda_{1}, \lambda_{2}, \lambda_{4}\right)}=g_{\left(\mu_{1}-2, \mu_{2}-2\right)\left(\nu_{1}-2, \nu_{2}-2\right)}^{\left(\lambda_{1}-1, \lambda_{2}-1, \lambda_{2}-1, \lambda_{4}-1\right)}$ reduce the study of $G_{2,2}$ to the study of the function

$$
\left(n, \gamma_{1}, \gamma_{2}, r, s\right) \mapsto g_{(n-r, r)(n-s, s)}^{\left(n-\gamma_{1}-\gamma_{2}, \gamma_{1}, \gamma_{2}\right)}
$$

\section{Murnaghan's Theorem and reduced Kronecker coefficients}

In this section we introduce Murnaghan's reduced Kronecker coefficients $\bar{g}_{\alpha, \beta}^{\gamma}$. They are integers indexed by triples of partitions closely related to the Kronecker coefficients. The Kronecker coefficients indexed by two two-row shapes will be re-obtained from the reduced Kronecker coefficients indexed by two one-row shapes (Section 3 ) which will be easy to describe (Theorem 4 and Section 4 .

The Jacobi-Trudi formula expresses the Schur functions as determinants in the complete sums $h_{k}$. When $\lambda$ has at most $k$ parts, it asserts that:

$$
s_{\lambda}=\operatorname{det}\left(h_{j-i+\lambda_{i}}\right)_{i, j=1, \ldots, k}
$$

(where $h_{k}=0$ when $k<0, h_{0}=1$ and $\lambda_{i}=0$ for $i$ greater than the length of $\lambda$.) 
This formula can also be applied in the case when $\lambda$ is not a partition, i.e. is not nondecreasing. The functions $s_{\lambda}$ obtained are either 0 , or Schur functions up to a sign.

Let $n$ be an integer and $\lambda$ a partition. Then $|\lambda|$ stands for the sum of the parts of $\lambda$ and for any integer $n$, we denote with $(n-|\lambda|, \lambda)$ the sequence $\left(n-|\lambda|, \lambda_{1}, \lambda_{2}, \ldots\right)$. This is a partition if and only if $n \geq|\lambda|+\lambda_{1}$. Last $\bar{\lambda}$ stands for the partition $\left(\lambda_{2}, \lambda_{3}, \ldots\right)$, which is obtained by removing the first part of $\lambda$.

Theorem 2 (Murnaghan (1938, 1955)) There exists a family of nonnegative integers $\left(\bar{g}_{\alpha, \beta}^{\gamma}\right)$ indexed by triples of partitions $(\alpha, \beta, \gamma)$ such that, for fixed partitions $\alpha$ and $\beta$, only finitely many terms $\bar{g}_{\alpha, \beta}^{\gamma}$ are non-zero, and for all $n \geq 0$,

$$
s_{(n-|\alpha|, \alpha)} * s_{(n-|\beta|, \beta)}=\sum_{\gamma} \bar{g}_{\alpha, \beta}^{\gamma} s_{(n-|\gamma|, \gamma)}
$$

Following Klyachko (2004), we call the coefficients $\bar{g}_{\alpha, \beta}^{\gamma}$ the reduced Kronecker coefficients. They are called extended Littlewood-Richardson numbers in Kirillov (2004) because of the following property, observed first in Murnaghan (1955) and proved in Littlewood (1958): if $\alpha, \beta$ and $\gamma$ are three partitions such that $|\gamma|=|\alpha|+|\beta|$ then $\bar{g}_{\alpha \beta}^{\gamma}=c_{\alpha \beta}^{\gamma}$.

Remark 3 It follows from Murnaghan's Theorem that for fixed partitions $\alpha, \beta$, $\gamma$, the sequence of Kronecker coefficients $g_{(n-|\alpha|, \alpha),(n-|\beta|, \beta)}^{(n-|\gamma|, \gamma)}$ (n big enough so that all three indices are partitions) is stationary with limit $\bar{g}_{\alpha \beta}^{\gamma}$.

\section{From reduced to non-reduced Kronecker coefficients}

In this section we give a formula that allows us to recover the Kronecker coefficients from the reduced Kronecker coefficients, and we apply it for the Kronecker coefficients indexed by two two-row shapes.

For any infinite sequence $u=\left(u_{1}, u_{2}, \ldots\right)$ and any positive integer $i$ we denote with $u^{\dagger i}$ the sequence obtained from $u$ by incrementing by 1 its $i-1$ first terms and removing its $i$-th term, that is: $u^{\dagger i}=$ $\left(u_{1}+1, u_{2}+1, \ldots, u_{i-1}+1, u_{i+1}, u_{i+2} \ldots\right)$. Partitions are identified with infinite sequences by appending trailing zeros. Under this identification, if $\lambda$ is a partition then so is $\lambda^{\dagger i}$ for all $i$.

Theorem 3 Let $\ell_{1}, \ell_{2}$ and $n$ be positive integers. Let $\lambda, \mu, \nu$ be partitions of $n$ such that $\mu$ has length at most $\ell_{1}$ and $\nu$ has length at most $\ell_{2}$. Then:

$$
g_{\mu \nu}^{\lambda}=\sum_{i=1}^{\ell_{1} \ell_{2}}(-1)^{i+1} \bar{g}_{\bar{\mu}, \bar{\nu}}^{\lambda^{\dagger i}}
$$

For $\ell_{1}=\ell_{2}=2$, Formula [2] applies as follows:

$$
g_{(n-r, r)(n-s, s)}^{\left(\lambda_{1}, \lambda_{2}, \lambda_{3}\right)}=\bar{g}_{(r)(s)}^{\left(\lambda_{2}, \lambda_{3}\right)}-\bar{g}_{(r)(s)}^{\left(\lambda_{1}+1, \lambda_{3}\right)}+\bar{g}_{(r)(s)}^{\left(\lambda_{1}+1, \lambda_{2}+1\right)}
$$

where $n=|\lambda|$, because the last expected summand $\bar{g}_{(r)(s)}^{\left(\lambda_{1}+1, \lambda_{2}+1, \lambda_{3}+1\right)}$ is always zero.

The reduced Kronecker coefficients that appear in this formula are all of the form $\bar{g}_{(r)(s)}^{\left(\gamma_{1}, \gamma_{2}\right)}$. These coefficients admit the following description, derived in Briand et al. (2008) from the description for the Kronecker coefficients indexed by two two-row shapes provided by Rosas (2001). An equivalent description for the reduced Kronecker coefficients indexed by two one-row shapes is given by Thibon (1991). 
Theorem 4 (Briand et al. (2008)) Let $r$, $s$ and $\gamma_{1} \geq \gamma_{2}$ be nonnegative integers and $h=\left(r, s, \gamma_{1}, \gamma_{2}\right)$. The reduced Kronecker coefficient $\bar{g}_{(r)(s)}^{\left(\gamma_{1}, \gamma_{2}\right)}$ counts the integral solutions to the system of inequalities $u_{i}(X, Y)+c_{i}(h) \geq 0$ for $i=0, \ldots, 6$, where:

$$
\begin{aligned}
& u_{0}(v)+c_{0}(h)=X-s \\
& u_{1}(v)+c_{1}(h)=X-r \\
& u_{2}(v)+c_{2}(h)=X+Y-r-s+\gamma_{1} \\
& u_{3}(v)+c_{3}(h)=Y \\
& \begin{array}{l}
u_{4}(v)+c_{4}(h)=Y-X+|\gamma| \\
u_{5}(v)+c_{5}(h)=-X-Y+r+s-\gamma_{2} \\
u_{6}(v)+c_{6}(h)=X-Y-\gamma_{1}
\end{array}
\end{aligned}
$$

In particular, the function $R:\left(r, s, \gamma_{1}, \gamma_{2}\right) \in \mathbb{Z}^{4} \mapsto \bar{g}_{(r)(s)}^{\left(\gamma_{1}, \gamma_{2}\right)}$ is vector partition-like.

Theorem 4 and Formula $(3)$ provide a piecewise quasipolynomial description for $G_{2,2}$ (see Remark 2). But the corresponding domains of quasipolynomiality obtained are neither closed, nor cones. The remainder of this work is devoted to correct this and obtain, still from Theorem 4 and Formula (3) a vector partition-like presentation for $G_{2,2}$.

The main tools are the Lemma 1, below, and an explicit vector partition-like presentation for the function $R$ (section 4) showing that the lemma applies.

Let $F_{0}, F_{1}, F_{2}$ be the linear maps from $\mathbb{R}^{5}$ to $\mathbb{R}^{4}$ that send $\left(n, r, s, \gamma_{1}, \gamma_{2}\right)$ to $\left(r, s, \gamma_{1}, \gamma_{2}\right),(r, s, n-$ $\left.\gamma_{1}-\gamma_{2}, \gamma_{2}\right),\left(r, s, n-\gamma_{1}-\gamma_{2}, \gamma_{1}\right)$ respectively. Let $T_{1}$ and $T_{2}$ be the translations in $\mathbb{R}^{4}$ of vector $v_{1}=(0,0,1,0)$ and $v_{2}=(0,0,1,1)$ respectively.

Let $\Delta$ (resp. $\Delta^{\prime}$ ) be the cone of $\mathbb{R}^{5}$ (resp. of $\mathbb{R}^{4}$ ) generated by all $\left(n, r, s, \gamma_{1}, \gamma_{2}\right) \in \mathbb{Z}^{5}$ (resp. all $\left.\left(r, s, \gamma_{1}, \gamma_{2}\right) \in \mathbb{Z}^{4}\right)$ such that the Kronecker coefficient $g_{(n-r, r)(n-s, s)}^{\left(n-\gamma_{1}-\gamma_{2}, \gamma_{1}, \gamma_{2}\right)}$ (resp. the reduced Kronecker coefficient $\bar{g}_{(r)(s)}^{\left(\gamma_{1}, \gamma_{2}\right)}$ is defined and positive. The explicit description of $\Delta$ is provided by Bravyi (2004) (see also the general approach by Klyachko (2004)). The cone $\Delta^{\prime}$ is the image of $\Delta$ under $F_{0}$.

For $x \in \mathbb{Z}^{5}$ set $\chi_{\Delta}(x)=1$ if $x \in \Delta$ and $\chi_{\Delta}(x)=0$ otherwise. Then we can rewrite Formula 3 as follows:

$$
G(x)=R \circ F_{0}(x)-\chi_{\Delta}(x) \cdot R \circ T_{1} \circ F_{1}(x)+\chi_{\Delta}(x) \cdot R \circ T_{2} \circ F_{2}(x)
$$

where $G(x)=G\left(n, r, s, \gamma_{1}, \gamma_{2}\right)=g_{(n-r, r)(n-s, s)}^{\left(n-\gamma_{1}-\gamma_{2}, \gamma_{1}, \gamma_{2}\right)}$ when $(n-r, r),(n-s, s),\left(n-\gamma_{1}-\gamma_{2}, \gamma_{1}, \gamma_{2}\right)$ are partitions, and $G\left(n, r, s, \gamma_{1}, \gamma_{2}\right)=0$ otherwise.

After Remark 1] Theorem 1 will be proved if we show that all three vector partition-like functions $R \circ F_{0}, \chi_{\Delta} \cdot R \circ T_{1} \circ F_{1}$ and $\chi_{\Delta} \cdot R \circ T_{2} \circ F_{2}$ admit presentations with the same cone: $\left(\Delta, \mathfrak{F}_{0}, Q_{0}\right)$, $\left(\Delta, \mathfrak{F}_{1}, Q_{1}\right)$ and $\left(\Delta, \mathfrak{F}_{2}, Q_{2}\right)$.

That $R \circ F_{0}$ admits a presentation $\left(\Delta, \mathfrak{F}_{0}, Q_{0}\right)$ is immediate because $F_{0}^{-1}\left(\Delta^{\prime}\right)=\Delta$. To show that $\chi_{\Delta} \cdot R \circ T_{1} \circ F_{1}$ and $\chi_{\Delta} \cdot R \circ T_{2} \circ F_{2}$ also admit presentations with cone $\Delta$ we will need to apply two times Lemma 1 below, with $p=5, q=4, C=\Delta, C^{\prime}=\Delta^{\prime}, \phi=R$ and $F=F_{i}, v=v_{i}$ for $i=1,2$.

Given subsets $A, B$ of $\mathbb{R}^{q}$ we denote with $A+B$ the set $\{a+b \mid a \in A, b \in B\}$. Given $v \in \mathbb{R}^{q}$ and $I$ subset of $\mathbb{R}$ we denote with $I v$ the set $\{x v \mid x \in I\}$.

Lemma 1 Let $\phi$ be a vector partition-like function on $\mathbb{Z}^{q}$ with presentation $\left(C^{\prime}, \mathfrak{F}^{\prime}, Q\right)$. Let $C$ be a convex rational polyhedral cone of $\mathbb{R}^{p}$ and $F$ an integral linear map from $\mathbb{R}^{p}$ onto $\mathbb{R}^{q}$. Let $v \in \mathbb{Z}^{q}$ and $T$ be the translation of $\mathbb{R}^{q}$ of vector $v$. Let $\mathfrak{F}$ be the fan subdividing $C \cap F^{-1}\left(C^{\prime}\right)$, whose cells are all sets of the form $C \cap F^{-1}\left(\sigma^{\prime}\right)$ for $\sigma^{\prime}$ cell of $\mathfrak{F}^{\prime}$.

Assume that the cone $C \cap F^{-1}\left(C^{\prime}\right)$ is full-dimensional in $\mathbb{R}^{p}$. Assume also that: 
(a) Whenever $H$ is a hyperplane separating two adjacent maximal cells $\sigma_{1}^{\prime}, \sigma_{2}^{\prime}$ of $\mathfrak{F}^{\prime}$ such that $F(C)$ is not included in $H+\mathbb{R}_{+} v$, the following holds: The quasipolynomials $Q_{\sigma_{1}^{\prime}}$ and $Q_{\sigma_{2}^{\prime}}$ coincide on the integral points of the strip $H+] 0 ; 1] v$.

(b) Whenever $H$ is a hyperplane containing a facet of $C^{\prime}$, such that $\mathbb{R}_{+} v+F(C)$ is not contained in the half-plane $H+C^{\prime}$, the following holds: For all maximal cells $\sigma^{\prime}$ of $\mathfrak{F}^{\prime}$ having a facet contained in $H$, the quasipolynomial $Q_{\sigma^{\prime}}$ vanishes on the integral points of the strip $\left.\left.H+\right] 0 ; 1\right] v$.

Then

(i) The function $\phi \circ T \circ F$ is zero on the integral points of the closure of $C \backslash F^{-1}\left(C^{\prime}\right)$.

(ii) If $C \cap F^{-1}\left(\sigma^{\prime}\right)$ is a maximal cell of $\mathfrak{F}$ (where $\sigma^{\prime}$ is a maximal cell of $\mathfrak{F}^{\prime}$ ) then $\phi \circ T \circ F$ and $Q_{\sigma} \circ T \circ F$ coincide on its integral points.

Applying the lemma as indicated requires a precise description of a presentation $\left(\Delta^{\prime}, \mathfrak{F}_{R}, Q_{R}\right)$ of $R$. The next section provides such a description.

\section{Formulas for the reduced Kronecker coefficients indexed by two one-row shapes}

Let $u_{i}$ and $c_{i}$, for $i=0,1, \ldots, 6$ be the integral linear forms defined in (4). After Brion and Vergne(1997), the function $\psi$ that associates to $y \in \mathbb{Z}^{7}$ the number of integral solutions of the system $u_{i}(X, Y)+y_{i} \geq 0$, $i=0, \ldots, 6$ is a vector partition function. In particular, it admits a very well-described vector partitionlike presentation $\left(C_{\psi}, \mathcal{F}_{\psi}, Q_{\psi}\right)$. The corresponding fan is the chamber complex of $\psi$, see Brion and Vergne (1997); Sturmfels (1995).

Remember (Theorem 4 that $R$ is the function that associates the reduced Kronecker coefficient $\bar{g}_{(r)(s)}^{\left(\gamma_{1}, \gamma_{2}\right)}$ to $\left(r, s, \gamma_{1}, \gamma_{2}\right) \in \mathbb{Z}^{4}$. Then $R=\psi \circ c$, where $c$ is the linear map from $\mathbb{R}^{4}$ to $\mathbb{R}^{7}$ that maps $h=\left(r, s, \gamma_{1}, \gamma_{2}\right)$ to $\left(c_{0}(h), c_{1}(h), \ldots, c_{6}(h)\right)$. Therefore, one obtains a very explicit vector partition-like presentation $\left(c^{-1}\left(C_{\psi}\right), \mathfrak{F}_{R}, Q_{R}\right)$ for $R$ by taking for $\mathfrak{F}_{R}$ the inverse image of $\mathfrak{F}_{\psi}$ under $c$, and for $Q_{R}$ the family of functions $Q_{R, c^{-1}(\sigma)}=Q_{\psi, \sigma} \circ c$ for $\sigma$ maximal cell of $\mathfrak{F}_{\psi}$. We present this description.

Let $h \in \mathbb{R}^{7}$. Denote with $\Pi(h)$ the set of real solutions of the system (4). For $i=0,1, \ldots, 6$, let $L_{i}(h)$ be the line with equation $a_{i} X+b_{i} Y+c_{i}(h)=0$ where $u_{i}(X, Y)=a_{i} X+b_{i} Y$.

For any three elements $i, j, k$ of $\{0,1, \ldots, 6\}$ define:

$$
f_{i j k}(h)=-\left|\begin{array}{ccc}
a_{i} & a_{j} & a_{k} \\
b_{i} & b_{j} & b_{k} \\
c_{i}(h) & c_{j}(h) & c_{k}(h)
\end{array}\right|
$$

Define also $f_{25}=\gamma_{1}-\gamma_{2}$ and $f_{46}=\gamma_{2}$. The linear form $f_{25}$ (resp. $f_{46}$ ) is proportional to $f_{25 k}$ for all $k \neq 2,5$ (resp.: to $f_{46 k}$ for all $k \neq 4,6$ ) and its vanishing is the condition for the two parallel lines $L_{2}$ and $L_{5}$ (resp. $L_{4}$ and $L_{6}$ ) to coincide.

- The cone $c^{-1}\left(C_{\psi}\right)$ is equal to the cone $\Delta^{\prime}$ introduced in Section 3 . It is defined by the system of linear inequalities:

$$
f_{145} \leq 0, \quad f_{045} \leq 0, \quad f_{356} \leq 0, \quad f_{035} \leq 0, \quad f_{135} \leq 0, \quad f_{25} \geq 0, \quad f_{46} \geq 0 .
$$




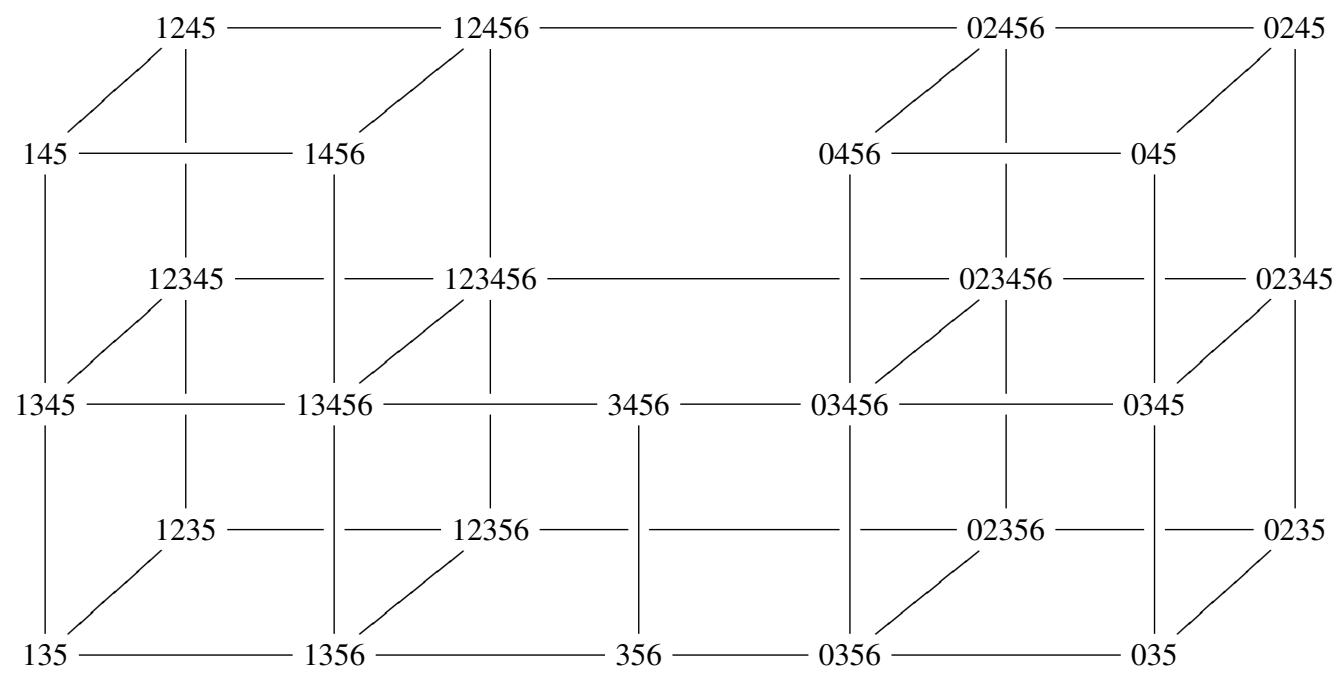

Fig. 1: The graph $\mathcal{G}$.

- The fan $\mathfrak{F}_{R}$ : Let $S$ be the locus of parameters $h$ such that three lines $L_{i}(h), L_{j}(h), L_{k}(h)$ meet in $\Pi(h)$. The fan $\mathfrak{F}_{R}$ is the fan whose chambers (maximal open cells) are the connected components of $\Delta^{\prime} \backslash S$. In each chamber $\sigma$ the set of indices $i$ such that $L_{i}(h)$ supports a side of $\Pi(h)$ is constant. Denote this set with $\operatorname{Sides}(\sigma)$. This set $\operatorname{Sides}(\sigma)$ determines $\sigma$. Therefore we denote a chamber $\sigma$ with $\sigma_{I}$ when $\operatorname{Sides}(\sigma)=I$, e.g. $\sigma_{1245}$ for the chamber $\sigma$ such that $\operatorname{Sides}(\sigma)=\{1,2,4,5\}$. There are 26 chambers $\sigma_{I}$ in $\mathfrak{F}_{R}$. The corresponding indices $I=\operatorname{Sides}\left(\sigma_{I}\right)$ are the vertices of the graph $\mathcal{G}$ in Figure 1] Adjacency in $\mathcal{G}$ represents adjacency in $\mathfrak{F}_{R}$ : chambers $\sigma_{I}$ and $\sigma_{J}$ are adjacent (i.e. their closures have a common facet) if and only if $I$ and $J$ are adjacent vertices in $\mathcal{G}$. Observe that when $\sigma_{I}$ and $\sigma_{J}$ are adjacent then:

- either $I$ and $J$ are obtained from each other by exchanging 0 and 1 . Then $\sigma_{I}$ and $\sigma_{J}$ are separated by the hyperplane of equation $r=s$. There is $r>s$ on $\sigma_{I}$ if $1 \in I$.

- or one of the sets is obtained from the other by inserting a unique element. Say $J=I \cup\{j\}$ with $j \notin I$. If the elements of $J$ are $p_{1}<p_{2}<\cdots<p_{t}$ say that the successor of $p_{q}$ is $p_{q+1}$, for $q=1, \ldots, t-1$, and that the successor of $p_{t}$ is $p_{1}$. This defines a cyclic order on $J$. Let $i$ and $k$ be the predecessor and successor of $j$ in this cyclic order. Then $\sigma_{I}$ and $\sigma_{J}$ are separated by the hyperplane of equation $f_{i j k}=0$, and $f_{i j k}>0$ on $\sigma_{I}$.

- The quasipolynomial formulas on each maximal cell: For simplicity we set $q_{I}=Q_{R, \overline{\sigma_{I}}}$. This is the quasipolynomial formula for $R$ valid on the cell $\overline{\sigma_{I}}$ (the topological closure of the chamber $\sigma_{I}$ ). Rather than displaying explicit expressions for all quasi-polynomials $q_{I}$, it is enough to present one of them (we choose $q_{135}$ ) and display all differences $q_{I}-q_{J}$ for $\sigma_{I}$ and $\sigma_{J}$ adjacent. All quasipolynomials $q_{I}$ can be recovered easily from this information by chasing on the graph $\mathcal{G}$ (Figure 1 , 


\begin{tabular}{|c|c|c|}
\hline$i j k$ & $q_{I}(h)-q_{J}(h)$ & $\begin{array}{c}\text { Values } \delta \text { s. t. } \\
q_{I}=q_{J} \\
\text { on } f_{i j k}=\delta \\
\end{array}$ \\
\hline $\begin{array}{l}613,123,134 \\
603,023,034\end{array}$ & $\frac{1}{2} f_{i j k}(h)\left(f_{i j k}(h)-1\right)$ & 0,1 \\
\hline 234 & $\frac{1}{4}\left(f_{i j k}(h)\right)^{2}+\left\{\begin{array}{cll}0 & \text { if } f_{i j k}(h) \equiv 0 & \bmod 2 \\
-1 / 4 & \text { else. }\end{array}\right.$ & $-1,0,1$ \\
\hline $\begin{array}{c}345,124,561 \\
024,560\end{array}$ & $\frac{1}{4} f_{i j k}(h)\left(f_{i j k}(h)-2\right)+\left\{\begin{array}{cll}0 & \text { if } f_{i j k}(h) \equiv 0 & \bmod 2 \\
1 / 4 & \text { else }\end{array}\right.$ & $0,1,2$ \\
\hline
\end{tabular}

Tab. 1: The differences $q_{I}-q_{J}$ for $\sigma_{I}$ and $\sigma_{J}$ adjacent chambers of $\mathfrak{F}$.

e.g.

$$
q_{1456}=\left(q_{1456}-q_{13456}\right)+\left(q_{13456}-q_{1356}\right)+\left(q_{1356}-q_{135}\right)+q_{135}
$$

There is:

$$
q_{135}\left(r, s, \gamma_{1}, \gamma_{2}\right)=\frac{1}{2}\left(s-\gamma_{2}+1\right)\left(s-\gamma_{2}+2\right)
$$

Let $\sigma_{I}$ and $\sigma_{J}$ be two adjacent chambers of $\mathfrak{F}$.

- If $I$ and $J$ are obtained from each other by exchanging 0 and 1 then $q_{I}=q_{J}$.

- If $J=I \cup\{j\}$ with $j \notin I$ then $q_{I}-q_{J}$ depend only of $j$ and its predecessor $i$ and successor $k$ in $J$, and is as indicated in Table 1 .

If $\sigma_{I}$ and $\sigma_{J}$ are adjacent, the quasi-polynomials $q_{I}$ and $q_{J}$ coincide not only on the affine hyperplane spanned by the facet $\overline{\sigma_{I}} \cap \overline{\sigma_{J}}$ but also on close parallel hyperplanes.

Proposition 1 Let $\sigma_{I}$ and $\sigma_{J}$ be two adjacent chambers of $\mathfrak{F}$ such that $J=I \cup\{j\}$ with $j \notin I$. Let $i$ and $k$ be the predecessor and successor, respectively, of $j$ in $J$.

Then $q_{I}-q_{J}$ coincide on the affine hyperplanes $f_{i j k}=\delta$ for the values of $\delta$ given by the third column in Table 1

Similarly, if the hyperplane $H$ supports a facet of a maximal cell $\overline{\sigma_{I}}$, and this facet is contained in the border of $\Delta^{\prime}$, then $q_{I}$ vanishes on affine hyperplanes close and parallel to $H$.

Proposition 2 Let $\sigma_{I}$ be a chamber of $\mathfrak{F}$ and $\tau$ an external facet of $\overline{\sigma_{I}}$ (i.e. a facet contained in the border of $\Delta^{\prime}$ ). The hyperplane supporting $\tau$ admits as equation $f=0$ where $f$ is one of the linear forms $f_{145}$, $f_{045}, f_{356}, f_{035}, f_{135}, f_{25}, f_{46}$.

The set of values $\delta \in \mathbb{Z}$ such that $f$ vanishes identically on the affine hyperplane of equation $f=\delta$ is provided by Table 2

It is immediate that $R \circ F_{0}$ has a vector partition-like presentations $\left(\Delta, \mathfrak{F}_{0}, Q_{0}\right)$. Propositions 1 and 2 are used to apply Lemma 1 and show that $\chi_{\Delta} \cdot R \circ T_{1} \circ F_{1}$ and $\chi_{\Delta} \cdot R \circ T_{2} \circ F_{2}$ have vector partition-like presentations $\left(\Delta, \mathfrak{F}_{1}, Q_{1}\right)$ and $\left(\Delta, \mathfrak{F}_{2}, Q_{2}\right)$. After Remark 1 , this proves Theorem 1 and provides a way to compute a vector partition-like presentation for $G$ and $G_{2,2}$. 


\begin{tabular}{|c|c|c|}
\hline Form $f$ & $\begin{array}{c}\text { Chambers having a facet } \\
\text { supported by } f=0\end{array}$ & $\begin{array}{c}\text { Values } \delta \text { such that } \\
q_{I} \text { vanishes identically } \\
\text { on } f=\delta\end{array}$ \\
\hline \hline$f_{46}=\gamma_{2}$ & $3456,1456,0456$ & -1 \\
\hline$f_{25}=\gamma_{1}-\gamma_{2}$ & $1245,0245,1235,0235$ & -1 \\
\hline$f_{145}=r-s-\gamma_{1}$ & 145 & $1,2,3$ \\
\hline$f_{045}=s-r-\gamma_{1}$ & 045 & $1,2,3$ \\
\hline$f_{356}=|\gamma|-r-s$ & 356 & $1,2,3$ \\
\hline$f_{035}=\gamma_{2}-r$ & 035 & 1,2 \\
\hline$f_{135}=\gamma_{2}-s$ & 135 & 1,2 \\
\hline
\end{tabular}

Tab. 2: The linear forms defining the facets of $\Delta^{\prime}$.

\section{Formulas for the Kronecker coefficients indexed by two two- row shapes}

Once the presentations $\left(\Delta, \mathfrak{F}_{0}, Q_{0}\right),\left(\Delta, \mathfrak{F}_{1}, Q_{1}\right),\left(\Delta, \mathfrak{F}_{2}, Q_{2}\right)$ for $R \circ F_{0}, \chi_{\Delta} \cdot R \circ T_{1} \circ F_{1}$ and $\chi_{\Delta} \cdot R \circ T_{2} \circ F_{2}$ have been determined, an explicit presentation $\left(\Delta, \mathfrak{F}_{3}, Q_{3}\right)$ for $G$ is obtained: The cells of $\mathfrak{F}_{3}$ are the intersection $\sigma_{0} \cap \sigma_{1} \cap \sigma_{2}$ for $\sigma_{i}$ a cell of $\mathfrak{F}_{i}, i \in\{0,1,2\}$. If $\sigma_{0} \cap \sigma_{1} \cap \sigma_{2}$ is a maximal cell of $\mathfrak{F}_{3}$ then the corresponding quasipolynomial formula for $G$ is $Q_{0, \sigma_{0}}-Q_{1, \sigma_{1}}+Q_{2, \sigma_{2}}$. We computed the description for $\mathfrak{F}_{3}$ by using the Maple Package CONVEX by Franz (2006): it has 177 maximal cells. It turns out that on some of them $G$ is given by the same quasipolynomial formulas, and that they can be glued together to form the maximal cells of a new fan $\mathfrak{F}_{K}$. In the new presentation $\left(\Delta, \mathfrak{F}_{K}, P\right)$ obtained for $G$ the fan $\mathfrak{F}_{K}$ has only 74 maximal cells.

All 74 quasipolynomial formulas $P_{\sigma}$ have the following form:

$$
P_{\sigma}=1 / 4 Q_{\sigma}+1 / 2 L_{\sigma}+M_{\sigma} / 4
$$

where $Q_{\sigma}$ and $L_{\sigma}$ are integral homogeneous polynomials in $\left(n, r, s, \gamma_{1}, \gamma_{2}\right)$ respectively quadratic and linear. The function $M_{\sigma}$ takes integral values, fulfills $M_{\sigma}(0) / 4=1$ and is constant on each coset of $\mathbb{Z}^{5}$ modulo the sublattice defined by $r+s \equiv n \equiv \gamma_{1} \equiv \gamma_{2} \equiv 0 \bmod 2$.

Moreover, for all maximal cells $\sigma$, the functions $Q_{\sigma}, L_{\sigma}$ are nonnegative on $\sigma$. This also holds for $M_{\sigma}$, for all cells $\sigma$ except four. This makes specially easy studying the support of the Kronecker coefficients indexed by two two-row shapes. This is the set of all triples $(\lambda, \mu, \nu)$ such that $g_{\mu, \nu}^{\lambda}>0$ and $\mu$ and $\nu$ have at most two parts.

We obtain the following result. Let $\left(n, r, s, \gamma_{1}, \gamma_{2}\right) \in \Delta$. Then $g_{(n-r, r)(n-s, s)}^{\left(n-\gamma_{1}-\gamma_{2}, \gamma_{1}, \gamma_{2}\right)}$ is zero if and only if at least one of the following five systems of conditions is fulfilled:

$$
\begin{aligned}
& \begin{array}{ll}
\left\{\begin{array}{l}
n s=2 r \\
\gamma_{1} \text { or } \gamma_{2} \text { odd. }
\end{array}\right. & \left\{\begin{array}{l}
n=\max \left(2 r, 2 s,|\gamma|+\gamma_{1}\right) \\
\gamma_{2}=0 \\
r+s+\gamma_{1} \text { odd. }
\end{array}\right. \\
\left\{\begin{array}{l}
n=\max (2 r, 2 s) \\
\gamma_{1}=\gamma_{2} \\
r+s+\gamma_{1} \text { odd. }
\end{array}\right. & \left\{\begin{array}{l}
n=|\gamma|+\gamma_{1}=\max (2 r, 2 s) \\
r+s+\gamma_{1} \text { odd. }
\end{array}\right.
\end{array}\left\{\begin{array}{l}
n=\max (2 r, 2 s) \\
|r-s|=1 \\
\min (2 r, 2 s) \geq|\gamma|+\gamma_{1} \\
\gamma_{1} \text { or } \gamma_{2} \text { even. }
\end{array}\right.
\end{aligned}
$$


This exhaustive description led us to a family of counterexamples for $\mathrm{SH}$, a saturation conjecture formulated by Mulmuley (2007). The stretching functions $\widetilde{g}_{\mu, \nu}^{\lambda}: N \mapsto g_{N \mu, N \nu}^{N \lambda}$ attached to the Kronecker coefficients are quasipolynomials (Mulmuley, 2007). This means that for any fixed $\lambda, \mu, \nu$ there exist an integer $k$ and polynomials $p_{1}, p_{2}, \ldots, p_{k}$ such that for any $N \geq 1, \widetilde{g}_{\mu, \nu}^{\lambda}(N)=p_{i}(N)$ when $N \equiv i$ $\bmod k$. Mulmuley's SH conjecture stated that for any such description, $g_{\mu, \nu}^{\lambda}=0 \Leftrightarrow F_{1}=0$. The rightmost system of conditions in (7) above provides a family of counterexamples to this conjecture (Briand et al. 2008). The discovery of these counterexamples led Mulmuley (2008) to propose a weaker form of the conjecture SH, still strong enough for the aims of Geometric Complexity Theory.

\section{References}

A. D. Berenstein and A. V. Zelevinsky. Triple multiplicities for $\operatorname{sl}(r+1)$ and the spectrum of the exterior algebra of the adjoint representation. J. Algebraic Combin., 1(1):7-22, 1992.

Sergey Bravyi. Requirements for compatibility between local and multipartite quantum states. Quantum Inf. Comput., 4(1):12-26, 2004.

Emmanuel Briand, Rosa Orellana, and Mercedes Rosas. Reduced Kronecker coefficients and counterexamples to Mulmuley's strong saturation conjecture SH. ArXiv:0810.3163v2, 2008. To appear in Comp. Complexity.

Emmanuel Briand, Rosa Orellana, and Mercedes Rosas. Quasi-polynomial formulas for the Kronecker coefficients indexed by two two-row shapes. In preparation.

Michel Brion and Michèle Vergne. Residue formulae, vector partition functions and lattice points in rational polytopes. J. Amer. Math. Soc., 10(4):797-833, 1997.

Andrew A.H. Brown, Stephanie Van Willigenburg, and Mike Zabrocki. Expressions for Catalan Kronecker products. ArXiv:0809.3469v1, 2008.

Anders Skovsted Buch. The saturation conjecture (after A. Knutson and T. Tao). Enseign. Math. (2), 46 (1-2):43-60, 2000. With an appendix by William Fulton.

Peter Bürgisser and Christian Ikenmeyer. The complexity of computing Kronecker coefficients. In Proceedings of FPSAC 2008 (Formal Power Series and Algebraic Combinatorics), Valparaiso, 2008.

Matthias Franz. Convex - a Maple package for convex geometry, version 1.1, 2006. available at http://www-fourier.ujf-grenoble.fr/ franz/convex/.

A.M. Garsia, G. Musiker, N. Wallach, and G. Xin. Invariants, Kronecker products, and combinatorics of some remarkable diophantine systems. ArXiv:0810.0060v1, 2008.

Anatol N. Kirillov. An invitation to the generalized saturation conjecture. Publ. Res. Inst. Math. Sci., 40 (4):1147-1239, 2004.

Alexander Klyachko. Quantum marginal problem and representations of the symmetric group. arXiv:quant-ph:0409113, september 2004. 
Allen Knutson and Terence Tao. The honeycomb model of $\mathrm{GL}_{n}(\mathbf{C})$ tensor products. I. Proof of the saturation conjecture. J. Amer. Math. Soc., 12(4):1055-1090, 1999.

D. E. Littlewood. Products and plethysms of characters with orthogonal, symplectic and symmetric groups. Canad. J. Math., 10:17-32, 1958.

Jean-Gabriel Luque and Jean-Yves Thibon. Polynomial invariants of four qubits. Phys. Rev. A (3), 67(4): 042303, 5, 2003.

Ketan D. Mulmuley and Milind Sohoni. Geometric complexity theory. I. An approach to the P vs. NP and related problems. SIAM J. Comput., 31(2):496-526 (electronic), 2001.

Ketan D. Mulmuley and Milind Sohoni. Geometric complexity theory III: on deciding positivity of Littlewood-Richardson coefficients. ArXiv:cs.CC/0501076, January 2005.

Ketan D. Mulmuley. Geometric complexity theory VI: the flip via saturated and positive integer programming in representation theory and algebraic geometry. Technical Report TR-2007-04, Computer Science Department, The University of Chicago, may 2007. Available as arXiv:0704.0229 and at http://ramakrishnadas.cs.uchicago.edu. Revised version to be available here.

Ketan D. Mulmuley. Erratum to the saturation hypothesis (SH) in "Geometric Complexity Theory VI". Technical report TR2008-10, Computer Science Department, University of Chicago, 2008. http: //ramakrishnadas.cs.uchicago.edu.

Francis D. Murnaghan. The Analysis of the Kronecker Product of Irreducible Representations of the Symmetric Group. Amer. J. Math., 60(3):761-784, 1938.

Francis D. Murnaghan. On the analysis of the Kronecker product of irreducible representations of $S_{n}$. Proc. Nat. Acad. Sci. U.S.A., 41:515-518, 1955.

Hariharan Narayanan. On the complexity of computing Kostka numbers and Littlewood-Richardson coefficients. Journal of Algebraic Combinatorics, 24(3):347-354, 2006.

Etienne Rassart. A polynomiality property for Littlewood-Richardson coefficients. J. Combin. Theory Ser. A, 107(2):161-179, 2004.

Jeffrey B. Remmel and Tamsen Whitehead. On the Kronecker product of Schur functions of two row shapes. Bull. Belg. Math. Soc. Simon Stevin, 1:649-683, 1994.

Mercedes H. Rosas. The Kronecker product of Schur functions indexed by two-row shapes or hook shapes. Journal of algebraic combinatorics, 14(2):153-173, 2001. Also at arXiv:math.CO/0001084.

Richard P. Stanley. Enumerative combinatorics. Vol. 1, volume 49 of Cambridge Studies in Advanced Mathematics. Cambridge University Press, Cambridge, 1997. With a foreword by Gian-Carlo Rota, Corrected reprint of the 1986 original.

Bernd Sturmfels. On vector partition functions. J. Combin. Theory Ser. A, 72(2):302-309, 1995. ISSN 0097-3165.

Jean-Yves Thibon. Hopf algebras of symmetric functions and tensor products of symmetric group representations. Internat. J. Algebra Comput., 1(2):207-221, 1991. 